\title{
BMJ Open Effect of glucomannan supplementation on body weight in overweight and obese children: protocol of a randomised controlled trial
}

\author{
Bartłomiej Mateusz Zalewski, Hania Szajewska
}

To cite: Zalewski BM, Szajewska $H$. Effect of glucomannan supplementation on body weight in overweight and obese children: protocol of a randomised controlled trial. BMJ Open 2015;5:e007244. doi:10.1136/bmjopen-2014007244

- Prepublication history for this paper is available online. To view these files please visit the journal online (http://dx.doi.org/10.1136/ bmjopen-2014-007244).

Received 19 November 2014 Revised 27 January 2015 Accepted 1 February 2015

\section{CrossMark}

Department of Paediatrics, The Medical University of Warsaw, Warsaw, Poland

Correspondence to Dr Bartłomiej Mateusz

Zalewski;

zalewski.bm@gmail.com

\begin{abstract}
Introduction: Glucomannan (GNN), a water-soluble dietary fibre derived from the plant Amorphophallus konjac, is marketed for weight reduction. The exact mechanisms by which GNN might exert its actions are unclear. However, it has been shown that GNN slows gastric emptying by forming a viscous gel of large volume, which increases the feeling of satiety. Current evidence on the effectiveness of GNN for weight reduction is sparse, and well-designed trials performed in children are needed to assess the efficacy of this modality. We aim to systematically evaluate the efficacy of GNN consumption for the management of children who are overweight or obese.
\end{abstract}

Methods and analysis: Children aged 6-17 years who are overweight or obese (based on the WHO growth criteria) will be randomly assigned to receive GNN or placebo (maltodextrin) (both at a dose of $3 \mathrm{~g} /$ day) for 3 months and will be followed-up for 3 months. Before the intervention, all children will receive dietetic advice, and they will be encouraged to engage in physical activity. The primary outcome measure will be the body mass index-for-age z-score difference between the groups at the end of the intervention.

Ethics and dissemination: The study was approved by the Bioethics Committee of the Medical University of Warsaw. The findings of this trial will be submitted to a peer-reviewed journal (paediatric, nutrition or gastroenterology). Abstracts will be submitted to relevant national and international conferences.

Trial registration number: NCT02280772.

\section{INTRODUCTION}

\section{Background and rationale}

Obesity is a major global health challenge, ${ }^{1}$ and there is continuous research directed at identifying interventions that will effectively help in body mass reduction. The current recommendations for weight management offer comprehensive lifestyle interventions, including counselling and education, aimed at reducing weight. ${ }^{2}$ However, in clinical

\section{Strengths and limitations of this study}

- This randomised, double-blind, placebocontrolled trial will help to resolve the uncertainty regarding the role, if any, of glucomannan (GNN), a water-soluble dietary fibre derived from the plant Amorphophallus konjac in the management of children who are overweight or obese, one of the most common problems worldwide.

- This study will be performed at a research centre with experience in conducting independent, investigator-initiated, randomised controlled trials.

- The study is a single-centre study. GNN is not available worldwide. The generalisability of the study findings will depend on the setting.

- The dosing of GNN is not clearly established.

There is no long-term follow-up.

practice, patients have difficulty complying with these lifestyle interventions. The lack of an effective standard care for overweight and obese children stimulates research for supportive modalities. $^{3}$

In many countries, glucomannan (GNN), a water-soluble dietary fibre derived from the plant Amorphophallus konjac, is marketed for weight reduction. The exact mechanisms by which GNN might exert its actions are unclear. However, it has been shown that GNN slows gastric emptying by forming a viscous gel of large volume, which increases the feeling of satiety. ${ }^{4}$

Recently, we carried out a systematic review of randomised controlled trials (RCTs) to evaluate the effects of GNN on body weight and body mass index (BMI) in otherwise healthy overweight or obese children and adults. Limited data suggest that, in the short term, GNN has the potential to reduce body weight, but not BMI, in adults. Data in children were too limited to allow any conclusions to be drawn. The overall quality of the trials was moderate, with small study groups and short intervention and follow-up times. 
Earlier systematic review, however, including only RCTs carried out in adults revealed a non-significant difference in weight loss between GNN and placebo groups. ${ }^{7}$ Considering that current evidence on the effectiveness of GNN in children is sparse, well-designed RCTs performed in children are needed to assess the efficacy of this modality.

\section{Study objective}

The aim of the study is to determine the effectiveness of GNN administration on body weight and BMI of overweight and obese children.

\section{METHODS}

\section{Study design/setting}

This study is designed as a parallel-group, superiority, randomised, double-blind, placebo-controlled, singlecentre trial, with allocation 1:1. The recruitment of the study subjects will take place in the Department of Paediatrics, The Medical University of Warsaw, Poland (academic hospital).

\section{Inclusion/exclusion criteria}

At randomisation, children eligible for the trial must comply with all of the following inclusion criteria:

- age 6-17 years;

- overweight or obese based on the WHO growth charts/references (>+1 SD or >+2 SD, respectively).

Exclusion criteria are as follows:

- drug therapy for a chronic disease (including drugs that influence appetite or body weight);

- type 1 or 2 diabetes;

- history of surgical treatment of obesity;

- participation in another programme for treating obesity during the project and/or 3 months before recruitment;

- secondary causes of obesity;

- pregnancy.

\section{Intervention}

A summary of the trial is presented in table 1. Eligible children will be randomly assigned to receive GNN or a comparable placebo (maltodextrin). Both products will be administered orally, at a dose of $3 \mathrm{~g} /$ day, for 3 months. Patients will be followed-up for the next 3 months to assess the retention of a possible effect.

The choice of maltodextrin as a placebo is based on the results of our systematic review and a previous RCT carried out by our team. ${ }^{7} 8$

The dose of GNN is based on the results of the systematic review by Sood $e t a l l^{9}$ which showed that a daily dose of 2-3 $\mathrm{g}$ was usually prescribed. In our systematic review, ${ }^{7}$ the only RCT performed in children showed no effect of GNN use ( $2 \mathrm{~g}$ /day) for 2 months. Therefore, we decided to use a dosage of GNN of $3 \mathrm{~g} /$ day (in three divided doses) for 12 weeks. The planned duration of the administration was based on the minimal time of an intervention for inclusion in the Cochrane Collaboration systematic review of interventions for treating obesity in children. ${ }^{10}$

Both GNN and the placebo will be manufactured and supplied by Dicofarm SpA (Rome, Italy) as capsules in identical packaging. The manufacturer had no role in the conception or design of the study, and will have no role in the conduct of the study, or in the analysis or interpretation of the data. The administration of study products will start after a consultation with a dietitian (within 1 week of the enrolment visit).

The research team will monitor the study for acceptance of the study products and adverse events. If needed, discontinuation or modification of the treatment may be considered at the discretion of the physician.

A face-to-face adherence discussion will take place at the initial visit and at each study visit thereafter, emphasising the importance of following study guidelines and the instructions about taking the study product. Participants will be asked to bring all remaining capsules to each visit. To enhance validity of the data, capsules will be counted at each study visit. We will calculate the percentage adherence to therapy, based on the number of capsules consumed versus anticipated capsule consumption.

Both groups will receive the same concomitant care.

1. Children and their caregivers will receive individually suited dietary advice based on the national daily allowances and physical activity levels. A dietitian will perform a qualitative and quantitative analysis of the child's food intake, based on a 3-day food record (over 2 week days and 1 weekend day). This will be then reviewed using the computer software DIETA 5.0; http://www.izz.waw.pl (2011, Warsaw, Poland). The Nutrition Standards for the Polish population will be used to calculate energy needs, considering the subject's age, sex and level of physical activity. ${ }^{11}$ No specific dietary plan, including calorie-restriction diets, will be prescribed. The consultation with a dietitian is planned at the beginning of the study, at week 12 , and at week 24.

2. All participants, at each programme visit, will be encouraged to be physically active (with a goal of more than $60 \mathrm{~min}$ a day of a moderate-to-vigorous physical activity). However, otherwise, no specific physical activity plan will be advised.

3. Children and parents will be advised to limit sedentary/screen time to $\leq 2 \mathrm{~h}$ a day. ${ }^{2}$

At entry, maturity stage will be assessed according to the criteria of Tanner for secondary sexual characteristics.

\section{Follow-up}

All study participants will be followed-up for the duration of the intervention (3 months) and then for an additional 3 months.

\section{Criteria for discontinuing interventions}

Participants may discontinue the trial at their request or on the occurrence of serious adverse events. 
Table 1 Summary of the trial

\begin{tabular}{|c|c|c|c|c|c|c|c|}
\hline \multirow[b]{2}{*}{ Time point } & \multirow[b]{2}{*}{ At enrolment } & \multirow{2}{*}{$\begin{array}{l}\text { Randomisation } \\
\text { Start }\end{array}$} & \multicolumn{5}{|c|}{ After allocation } \\
\hline & & & Week 6 & Week 12 & Week 13 & Week 18 & Week 24 \\
\hline \multicolumn{8}{|l|}{ Enrolment } \\
\hline Eligibility screen & + & & & & & & \\
\hline Informed consent & + & & & & & & \\
\hline $\begin{array}{l}\text { Maturity stage (the criteria of } \\
\text { Tanner) }\end{array}$ & + & & & + & & & + \\
\hline Randomisation & & + & & & & & \\
\hline Product dispensation & & + & + & & & & \\
\hline Interventions (week 0-week 12) & & + & + & + & & & \\
\hline Follow-up (week 13-week 24) & & & & & + & + & + \\
\hline \multicolumn{8}{|l|}{ Assessments } \\
\hline Anthropometry & + & + & + & + & & + & + \\
\hline $\begin{array}{l}\text { Body composition (DXA } \\
\text { measurement) }\end{array}$ & + & & & & + & & \\
\hline 3-day food record & + & & & + & & & + \\
\hline Dietitian's assessment & + & & & + & & & + \\
\hline Physical activity assessment & + & & & + & & & \\
\hline $\begin{array}{l}\text { Lipids and fasting plasma } \\
\text { glucose }\end{array}$ & + & & & + & & & + \\
\hline Blood pressure measurement & + & & + & + & & + & + \\
\hline Return of unused study products & & & + & + & & & \\
\hline Adverse events & & + & + & + & & & \\
\hline
\end{tabular}

\section{Outcomes}

The primary outcome measure will be the BMI-for-age z-score difference (baseline vs end of the intervention) between the GNN and placebo groups at 12 weeks. According to Must and Anderson, ${ }^{12}$ this measure can be appropriately used as a comparison between group means and as a model of longitudinal weight trajectories.

BMI will be computed by dividing weight $(\mathrm{kg})$ by height squared $\left(\mathrm{m}^{2}\right)$. The BMI-for-age $\mathrm{z}$-score is the number of SDs by which the BMI in a child differs from the mean BMI of children of the same age and gender. It will be computed using the WHO AnthroPlus software V.1.04. Body weight and height measurements will be obtained at the hospital at every study visit. Body weight ( $\mathrm{kg}$ ) will be measured using the Radwag digital scale to the nearest $0.1 \mathrm{~kg}$ without shoes, in light indoor clothing. Standing height $(\mathrm{cm})$ will be measured using a Holtain stadiometer, to the nearest $0.1 \mathrm{~cm}$, barefoot and the head positioned in the Frankfurt horizontal plane. Before all measurements, we will ask the participants to visit a toilet.

The secondary outcome measures will include the following.

- Body composition. Whole body fat, central body fat, fat-free mass $(g)$.

- This outcome will be assessed with dual-energy X-ray absorption (DXA) technology, which is a valid and reliable methodology for quantifying body fat. ${ }^{13}$ Participants will be positioned on the scanner table using standard procedures, and total body cuts will be positioned as per standard manufacturer specifications. DXA scans will be performed at the baseline visit, and after the intervention period (week 13), using the Lunar Prodigy (GE Healthcare, Little Chalfont, Buckinghamshire, UK) in the Department of Medical Imaging at the Children's Memorial Health Institute.

- Change in BMI-for-age z-score between 0 and 24 weeks.

- Proportion of participants with dyslipidaemia from baseline to week 12 and week 24 (mean change, SD).

- Proportion of participants with impaired fasting plasma glucose (FPG), from baseline to week 12 and week 24 (mean change, SD).

- Lipids (total cholesterol, high-density lipoprotein cholesterol, low-density lipoprotein cholesterol and triglycerides in $\mathrm{mg} / \mathrm{dL}$ ) and FPG will be obtained at the baseline visit, at week 12, and at week 24 after an overnight fast in all participants with the use of standard methods at the hospital laboratory of the Medical University of Warsaw.

- Blood pressure (systolic and diastolic) (mean change, SD)

- Three individual blood pressure measurements $(\mathrm{mm} \mathrm{Hg})$ and pulse rates (beats/min) will be taken in the sitting position on the right hand side according to the accepted standards at each study visit using an automatic oscillometer. ${ }^{14}$ Briefly, two readings will be taken at intervals of at least $1 \mathrm{~min}$, and the average of those readings will be used for analysis. However, when the difference between the first and second reading is $\geq 5 \mathrm{~mm} \mathrm{Hg}$, additional (one or two) readings will be obtained. The mean change from baseline to week 12 and week 24 will be calculated as an outcome. 
- Energy intake (kJ/day) at baseline and at week 12 and week 24.

- Assessment will be based on self-written, 3-day food records (reviewed by a dietitian using the computer software DIETA 5.0; http://www.izz.waw.pl (2011, Warsaw, Poland)).

- Physical activity (h/week) at baseline and at week 12.

- Physical activity will be assessed using an accelerometer (Actigraph wGT3X-BT). Data will be analysed using Actilife software (V.6; Actigraph).

- Adverse events.

- Participants will be encouraged to report all possible adverse outcomes).

\section{Sample size calculation}

The primary end point is the difference in BMI-for-age z-scores between groups. Considering data from the literature ${ }^{15}$ we assumed that the mean difference (MD) would be the value 0.17 , with an SD of 0.267 . To detect this difference, with a power of $80 \%$ and a significance level of $5 \%$ and taking into account that $20 \%$ of the patients will be lost to follow-up, we calculated that 48 patients will be needed to be studied in each group. The sample size calculation was performed using StatsDirect statistical software (http://www.statsdirect.com. England: StatsDirect Ltd 2013). To assist in achieving this sample size, participants will be offered flexible appointment times.

\section{Recruitment}

Participants will be recruited at the Department of Paediatrics. We will advertise our study among primary care physicians, targeting healthcare providers.

Our team has conducted a similar RCT, which enrolled 97 children over 23 months. ${ }^{8}$ As the inclusion criteria are similar, we estimate 2 years will be sufficient for patient enrolment, and a further 3 months for data analysis. No incentives will be provided for study enrolment.

\section{Sequence generation}

Participants will be randomly assigned to either GNN or placebo groups with a 1:1 allocation by using a computergenerated randomisation schedule stratified by gender and age (6-11 years, middle childhood; 12-17 years, early adolescence) using permuted blocks of random sizes (the block size will be concealed until the end of the study). ${ }^{16}$ The randomisation list will be developed by an independent investigator with no clinical involvement in the conduct of the trial.

\section{Allocation concealment}

Allocation concealment will be ensured using opaque, sealed, numbered envelopes. The study products will be weighed, packaged and signed by consecutive numbers according to the randomisation list by the hospital pharmacy at the Medical University of Warsaw by independent personnel not involved in the conduct of the trial. The randomisation sequence and codes will be secured until all participants have been recruited into the trial and all data have been analysed.

\section{Blinding}

The study products (GNN and placebo) will be identically packaged capsules. All participants and investigators will be blinded to the assigned treatment throughout the study. Unblinding will occur after the final data analysis.

\section{Statistical analysis}

All analysis will be conducted on an intention-to-treat basis, including all patients in the groups to which they are randomised for whom outcomes will be available (including dropouts and withdrawals). Descriptive statistics will be used to summarise baseline characteristics. The Student $t$ test will be used to compare mean values of continuous variables approximating a normal distribution. For non-normally distributed variables, the MannWhitney $U$ test will be used. The $\chi^{2}$ test or Fisher exact test will be used, as appropriate, to compare percentages. The same computer software will be used to calculate the relative risk (RR), number needed to treat and MD, all with a $95 \%$ CI. The difference between study groups will be considered significant when the $\mathrm{p}$ value is $<0.05$, when the $95 \%$ CI for RR does not include 1.0 , or when the $95 \%$ CI for MD does not include 0. All statistical tests will be two tailed and performed at the 5\% level of significance.

\section{Methods for additional analyses}

Both the primary and secondary outcomes will be analysed using analysis of covariance (adjusted for the baseline value). Potential effect modification (confounding) due to any chance imbalance in pubertal status, age and sex will be counteracted by inclusion of these in a secondary analysis, using a multifactorial regression analysis.

\section{Missing data}

Every effort will be made to minimise missing baseline and outcome data. The amount of missing data will be reported for each randomised arm. If necessary, multiple imputation or Bayesian methods for missing data will be used as appropriate to address any missing data. ${ }^{17} 18$

\section{Ethics}

The study protocol and template consent forms have been reviewed and approved by the Bioethics Committee of the Medical University of Warsaw. An informed written consent form will be signed by a parent or legal guardian (and patients $\geq 16$ years) before the study enrolment. Any modifications to the protocol, which may affect the conduct of the study, potential benefits to the patients, or patient safety-including changes to the study design - will be reported to the ethics committee for all necessary amendments. All study-related information will be stored securely at the 
study site in locked cabinets, in an area with limited access (databases will be secured with a passwordprotected access system).

\section{Dissemination}

The findings of this RCT will be submitted to a peerreviewed journal (paediatric, nutrition or gastroenterology). Abstracts will be submitted to relevant national and international conferences. The results of the study will be available to the participants on request during a face-to-face meeting.

\section{CONCLUSIONS}

The effectiveness of GNN for the management of overweight and obesity in children is still under discussion. A definitive answer has not yet been provided. Our study, carried out at a research centre with experience in conducting independent, investigator-initiated RCTs, intends to address a gap in the field and will test the effectiveness of GNN for reducing body weight in overweight and obese children.

Contributors HS conceptualised the study. Both authors contributed to the design of the study and read and approved the manuscript. BMZ developed the first draft of the manuscript. Both authors contributed to the development of the study protocol and approved the final draft of the manuscript.

Funding This research will be fully funded by the Medical University of Warsaw.

Competing interests HS has participated as a speaker for Dicopharm, a manufacturer of GNN.

Ethics approval Bioethics Committee of The Medical University of Warsaw.

Provenance and peer review Not commissioned; externally peer reviewed.

Open Access This is an Open Access article distributed in accordance with the Creative Commons Attribution Non Commercial (CC BY-NC 4.0) license, which permits others to distribute, remix, adapt, build upon this work noncommercially, and license their derivative works on different terms, provided the original work is properly cited and the use is non-commercial. See: http:// creativecommons.org/licenses/by-nc/4.0/

\section{REFERENCES}

1. $\mathrm{Ng} \mathrm{M}$, Fleming $\mathrm{T}$, Robinson $\mathrm{M}$, et al. Global, regional, and national prevalence of overweight and obesity in children and adults during 1980-2013: a systematic analysis for the Global Burden of Disease Study 2013. Lancet 2014;384:766-81. [Published Online First 29 May 2014].

2. Expert Panel on Integrated Guidelines for Cardiovascular Health and Risk Reduction in Children and Adolescents; National Heart, Lung, and Blood Institute. Expert panel on integrated guidelines for cardiovascular health and risk reduction in children and adolescents: summary report.
Pediatrics 2011;128(Suppl 5):S213-56. [Published Online First 14 Nov 2011].

3. Kalavainen M, Korppi M, Nuutinen O. Long-term efficacy of group-based treatment for childhood obesity compared with routinely given individual counselling. Int $J$ Obes (Lond) 2011;35:530-3. [Published Online First 1 Feb 2011].

4. Keithley J, Swanson B. Glucomannan and obesity: a critical review. Altern Ther Health MedAlternative Therapies in Health and Medicine 2005;11:30-344.

5. EFSA Panel on Dietetic Products NaAN. Scientific Opinion on the substantiation of health claims related to konjac mannan (glucomannan) and reduction of body weight (ID 854, 1556, 3725), reduction of post-prandial glycaemic responses (ID 1559), maintenance of normal blood glucose concentrations (ID 835, 3724), maintenance of normal (fasting) blood concentrations of triglycerides (ID 3217), maintenance of normal blood cholesterol concentrations (ID 3100, 3217), maintenance of normal bowel function (ID 834, $1557,3901)$ and decreasing potentially pathogenic gastro-intestinal microorganisms (ID 1558) pursuant to Article 13(1) of Regulation (EC) No 1924/2006. EFSA Journal 2010;8:1798.

6. Zalewski BM, Chmielewska A, Szajewska H. The effect of glucomannan on body weight in overweight or obese children and adults: a systematic review of randomized controlled trials. Nutrition 2015;31:437-442.e2. Published Online First 8 Oct 2014.

7. Onakpoya I, Posadzki P, Ernst E. The efficacy of glucomannan supplementation in overweight and obesity: a systematic review and meta-analysis of randomized clinical trials. J Am Coll Nutr 2014;33:70-8. [Published Online First 17 Feb 2014].

8. Liber A, Szajewska $\mathrm{H}$. Effect of oligofructose supplementation on body weight in overweight and obese children: a randomised, double-blind, placebo-controlled trial. Br J Nutr 2014;112:2068-74. [Published Online First 20 Oct 2014].

9. Sood N, Baker WL, Coleman Cl. Effect of glucomannan on plasma lipid and glucose concentrations, body weight, and blood pressure: systematic review and meta-analysis. Am J Clin NutrAmerican Journal of Clinical Nutrition 2008;88:1167-75.

10. Oude Luttikhuis $\mathrm{H}$, Baur L, Jansen $\mathrm{H}$, et al. Interventions for treating obesity in children. Cochrane Database Syst Rev 2009;(1): CD001872. [Published Online First 20 Jan 2009].

11. Jarosz M. Normy żywienia człowieka dla populacji polskiejnowelizacja. [Human nutrition standards for the Polish populationamendment]. Instytut Zywnosci i Zywienia. 2012.

12. Must A, Anderson SE. Body mass index in children and adolescents: considerations for population-based applications. Int $\mathrm{J}$ Obes (Lond) 2006;30:590-4.

13. Bridge $P$, Pocock NA, Nguyen $T$, et al. Prediction of appendicular skeletal and fat mass in children: excellent concordance of dual-energy $\mathrm{X}$-ray absorptiometry and magnetic resonance imaging. $J$ Pediatr Endocrinol Metab 2009;22:795-804.

14. Pickering TG, Hall JE, Appel LJ, et al. Recommendations for blood pressure measurement in humans and experimental animals: part 1: blood pressure measurement in humans: a statement for professionals from the Subcommittee of Professional and Public Education of the American Heart Association Council on High Blood Pressure Research. Circulation 2005;8:697-716.

15. Ford AL, Bergh C, Sodersten P, et al. Treatment of childhood obesity by retraining eating behaviour: randomised controlled trial. BMJ 2010;340:b5388.

16. Williams K, Thomson D, Seto I, et al. Standard 6: age groups for pediatric trials. Pediatrics 2012;129:S153-60.

17. Sterne JA, White IR, Carlin JB, et al. Multiple imputation for missing data in epidemiological and clinical research: potential and pitfalls. BMJ 2009;338:b2393.

18. Daniels MJ, Hogan JW, eds. Missing data in longitudinal studies: strategies for Bayesian modeling and sensitivity analysis. Chapman and Hall, 2008. 